\title{
Discovery of Sun's Bharat Radiation emission causing Extreme Ultraviolet (EUV) and UV dominant optical radiation
}

\author{
M.A. Padmanabha Rao, PhD (AIIMS) \\ Former Professor of Medical Physics 114 Charak Sadan, Vikas Puri, New Delhi 110018, India,
}

\begin{abstract}
Existence of Bharat Radiation emission has been first predicted in 1998 to explain the experimental discovery of $U V$ dominant optical emission from radioisotopes and XRF (X-ray fluorescent) sources by a previously unknown atomic phenomenon. Presence of Bharat Radiation wavelengths has been shown for the first time in the wavelength gap in electromagnetic spectrum situated in between X-ray and the extreme ultraviolet (EUV) wavelengths in the revised $R b$ XRF spectrum. For evidence of Bharat Radiation, $a$ comparison of the solar spectrum reported by various researchers since 1960 with the revised Rb XRF spectrum met with unexpected success. The three distinct solar spectral ranges remained unrecognized for the last half a century are now identified as of X-rays up to $12.87 \mathrm{~nm}$, Bharat Radiation from 12.87 to $31 \mathrm{~nm}$, and EUV from $31 \mathrm{~nm}$ onwards. Evidence of Sun's Bharat Radiation emission implies a fundamental change in our understanding the Sunlight phenomenon from the traditional belief that fusion powers Sun light. This new insight supports the previously reported author's view that $\gamma$-, $X$-, and $\beta$ emissions from fission products (radioisotopes) of ${ }^{235} U$ fission taking place on Sun's visible surface cause Bharat Radiation followed by EUV and UV dominant optical emission.
\end{abstract}

Key words: atomic phenomenon, Bharat Radiation, Coronal radioisotopic ions, Extreme ultraviolet (EUV), EVE suite, Flare emission lines, Radioisotopes, Rb X-ray spectrum, XRF sources, Solar XUV spectrum, Solar Xrays, Uranium fission, UV dominant optical emission, MEGS

\section{Introduction}

UV dominant optical emission experimentally detected by the author for the first time from radioisotopes and XRF sources could be successfully explained by a previously unknown atomic phenomenon [1- 6]. The $\gamma-, X-$, or $\beta$ energy causes Bharat Radiation with energy at eV level but higher than that of UV within the same excited atom of a radioisotope or XRF source, while passing through core-Coulomb space. Bharat Radiation, in turn causes UV dominant optical emission on valence excitation. That is how Bharat Radiation came into existence since its first prediction in 1998 [1].

A search for evidence of Bharat Radiation wavelengths in solar spectra reported by various researchers since 1960s met with unexpected success [7-11]. Since the inception of solar spectroscopy three distinct wavelength ranges looking like mounts covering the entire solar spectrum remained puzzling for being unclear what each one of the three mounts represent. The Bharat Radiation wavelengths, predicted from radioisotopes and XRF sources were already detected as a mount since long in the middle of solar spectrum. However, it remained a mystery what these wavelengths represent and their source. Eventually, interpretation of solar spectrum became possible only after the discovery of two more emissions, Bharat Radiation followed by UV dominant optical emission from radioisotopes and XRF sources. The three mount like wavelength ranges are now identified as of X-rays up to $12.87 \mathrm{~nm}$, Bharat Radiation from 12.87 to $31 \mathrm{~nm}$, and EUV beyond $31 \mathrm{~nm}$. The first and definite evidence of Bharat Radiation wavelengths in solar spectrum reported in this brief communication might signal a radical change in our current understanding on Sunlight phenomenon.

Solar spectral line emissions are so far believed to be the familiar atomic spectra of coronal ions at high temperatures. However, the presence of Bharat Radiation wavelengths in solar spectrum holds the key as they were caused by $\gamma-, X-$, and $\beta$ energies due to likely presence of radioisotopic ions on solar visible surface. This new insight supports the author's view that $\gamma-, X$-, and $\beta$ emissions from fission products (radioisotopes) of ${ }^{235} \mathrm{U}$ fission taking place on Sun's core surface cause Bharat Radiation followed by EUV and UV dominant optical emission [6]. This newly emerged nature of solar spectrum and the phenomenon involved in causing ultimately the EUV led to an understanding that solar line emissions are not caused by thermal excitation of coronal ions against the traditional wisdom. In fact, Bharat Radiation internally produced within an excited atom causes the new class of room temperature atomic spectra of solid radioisotopes on non-thermal valence excitation [6,12]. Hence solar flare line emissions seem to represent the new class of room temperature atomic spectra of solid radioisotopes caused by the same atomic phenomenon reportedly taking place in radioisotopes and XRF sources $[4,6,13]$. The reason why Bharat Radiation is able to cause EUV regardless of temperature is by virtue of its energy higher than that of EUV at eV level. The sequence of events namely $\gamma-, X-$, or $\beta$ energy causing Bharat 
Radiation in turn causing solar flare EUV, UV, visible, and near infrared radiation emissions implies that they all arise from coronal radioisotopic ions.

\section{Results and discussion}

Since Bharat Radiation wavelengths lie next to that of X-rays in the revised Rb XRF spectrum [6] reproduced in Figure 1 (a), it became necessary to find evidence of X-ray range in solar spectrum as the first step. There are reasons why X-ray range in solar spectrum remained elusive from previous researchers. For example, Woods et al. [14] suggested the following subdivisions: extreme ultraviolet (EUV) from 30 to $120 \mathrm{~nm}$, $\mathrm{X}$-ray ultraviolet (XUV) from 1 to $30 \mathrm{~nm}$, and $\mathrm{X}$ rays at wavelengths less than $1 \mathrm{~nm}$. Widely differing from this, Neupert [15] and Lilensten et al. [16] believed solar X-ray range would be below $10.0 \mathrm{~nm}$ in complete disagreement with the basic Rb X-ray spectrum [17], which ends at $12.87 \mathrm{~nm}$ as shown in the revised spectrum in Figure 1 (a). In reality, the solar spectra obtained by Hinteregger et al. in 1960 in Figure 1(b) displayed an intense spectral range on the left side that abruptly fell in intensity near $16.5 \mathrm{~nm}$ disagreeing with $12.87 \mathrm{~nm}$ in Figure 1 (a) [7 to 10]. Not able to understand what the intense spectral range represents, Friedman simply brushed away as an instrumental error [9]. Despite low resolution, with poor sensitivity and high background of the PMT (LiF cathode) used in their study the intensity fall at $16.5 \mathrm{~nm}$ being somewhat close to the end of $\mathrm{Rb}$ $\mathrm{X}$-ray range $12.87 \mathrm{~nm}$ raised hope that wavelengths shortward of $16.5 \mathrm{~nm}$ might represent solar X-ray range.

Hinteregger et al. (1960) suggested 30.0 to $91.0 \mathrm{~nm}$ as EUV range [8]. Hence the search for evidence of Bharat Radiation range has been confined in between X-ray $(16.5 \mathrm{~nm})$ and EUV (30.nm) ranges in Figure 1 (b). The current scenario is Herbert Friedman [9] opined wavelengths down to $20.0 \mathrm{~nm}$ as UV, and longward of $13.0 \mathrm{~nm}$ as EUV by Testa et al. [18] contrary to what has been actually observed in the solar spectrum. In Figure 1 (b), the intense spectral range started around $16.5 \mathrm{~nm}$ and ended with tallest peak at around $30.4 \mathrm{~nm}$ is in good agreement with the expected range 16.5 to $30.0 \mathrm{~nm}$ strongly suggesting the presence of Bharat Radiation wavelengths in solar spectrum. At this stage, difficulty arose in deciding whether the peak at $30.4 \mathrm{~nm}$ belongs to Bharat Radiation or EUV since Hinteregger et al. [8] suggested EUV begins at $30.0 \mathrm{~nm}$. Finally, spectrum obtained by Woods et al. [11] in Figure 3 resolved that the peak at $30.4 \mathrm{~nm}$ comes under Bharat Radiation. Therefore, in Figure 1 (b), wavelengths immediately after the peak at $30.4 \mathrm{~nm}$ are considered as EUV.

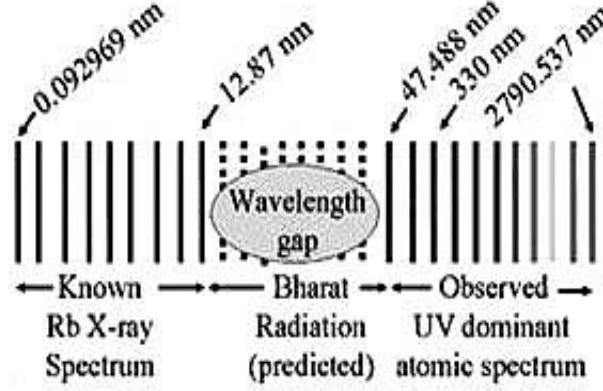

a

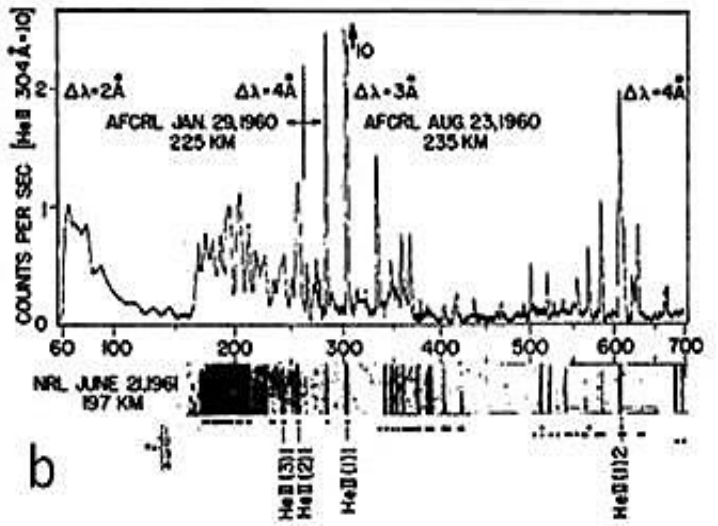

Fig.1. (a): Schematic of revised spectrum of Rb XRF source shows $12.87 \mathrm{~nm}$ is the end of familiar X-ray range and $47.488 \mathrm{~nm}$ [17] the beginning of EUV and the newly detected UV dominant optical range, so Bharat Radiation range was predicted to be somewhere between 12.87 to $47.488 \mathrm{~nm}$. (b): In 1960, Hinteregger et al. $[7,8,10]$ obtained two solar spectra on January 29 at $225 \mathrm{~km}$ height and August 23 at $235 \mathrm{~km}$ height and combined into a single XUV spectrum from 60 to $700 \AA$.

The spectra in Figure 2 were also obtained by Hinteregger et al. (1964) on 29 January 1960 at 103, 120 , 134, 147, 170 and $198 \mathrm{~km}$ heights lower than that in Figure 1 (b). The right intense spectral range gradually falling to base level around $13.0 \mathrm{~nm}$ signify a better agreement with the end of basic Rb XRF spectrum [17] at $12.87 \mathrm{~nm}$ in Figure 1(a) than the previous solar spectra in Figure 1(b). Thus Figure 2 provided the first key evidence that the wavelengths shortward of $13.0 \mathrm{~nm}$ represent X-ray range in solar spectrum but investigation continued for further confirmation and better results. 


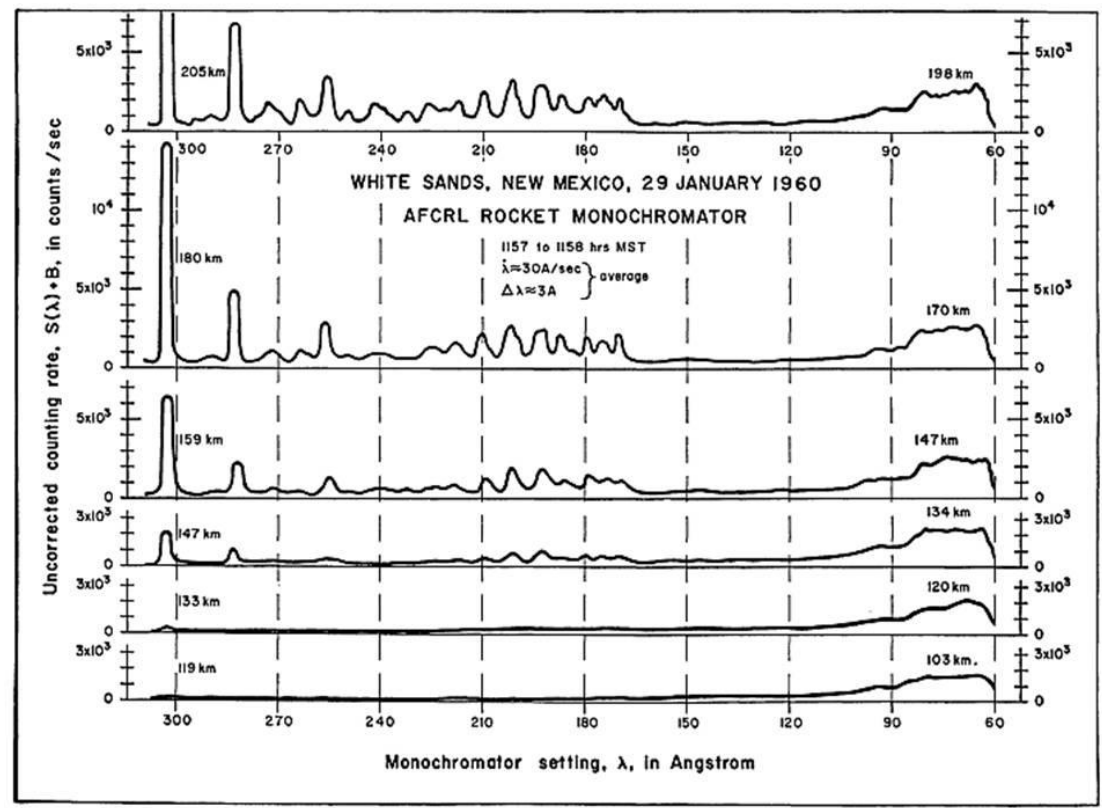

Fig 2. Solar XUV spectra that Hinteregger et al. [10] obtained at 103, 120, 134, 147, 170 and $198 \mathrm{~km}$ heights, lower than that shown in Figure 1(b) over New Mexico on 29 January 1960.

Next to the newly identified X-ray range, the solar spectrum at height $198 \mathrm{~km}$ showed intense spectral range beginning at around $16.5 \mathrm{~nm}$ and ending with the most intense peak at $30.4 \mathrm{~nm}$ as in the case of Figure 1 (b) strengthening the view on presence of Bharat Radiation range in solar spectrum. However, the investigation continued for further confirmation and better results.

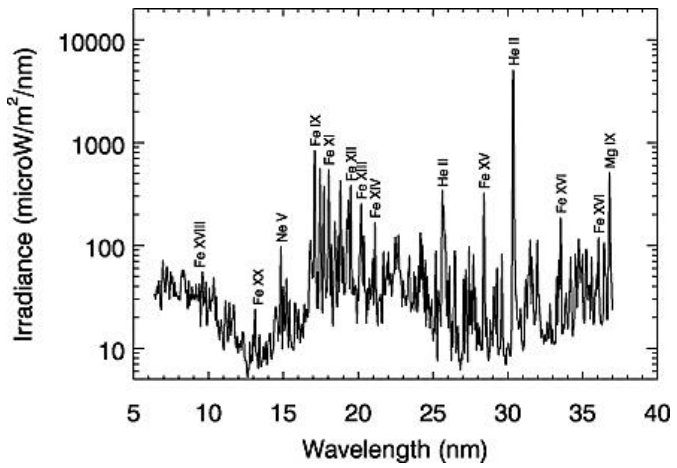

Fig.3. Solar spectrum shown here was obtained by Woods et al. [11] on May 5, 2010. The flare data from EVE shown here are primarily from the MEGS-A channel measuring the spectrum from 7 to $37 \mathrm{~nm}$.

As compared to solar spectra in Figures 1(b) and 2, the spectrum obtained by Woods et al. [11] in Figure 3 showed remarkable features: three intense spectral ranges adjacent to each other. This could be owing to the EVE suite including MEGS that has provided EUV spectral observations with spectral resolutions of 0.1 $\mathrm{nm}$, while the EUV SpectroPhotometer (ESP) provided broadband observations between 0.1 and $39 \mathrm{~nm}$ with an even higher cadence of $0.25 \mathrm{~s}$. On the basis of spectra in Figures 1 (b) and 2, the first intense spectral range on the left side is believed to be X-ray range. On calibration of solar spectrum with the spectrum of revised $\mathrm{Rb}$ XRF source, the minimum solar X-ray wavelength, $0.1 \mathrm{~nm}$ measured by ESP excellently agreed with 0.092969 $\mathrm{nm}\left({ }_{\mathrm{a} 1} \mathrm{KL}_{\mathrm{II}}\right)$ of basic $\mathrm{Rb} \mathrm{X}$-ray spectrum [6, 17] in Figure 1(a). Secondly, the spectral range sharply dipped to base level between 12 to $13 \mathrm{~nm}$ excellently agreeing with the end limit $12.87 \mathrm{~nm}$ of Rb X-ray spectrum. Thus these two evidences doubly ensured the first and direct evidence on the presence of X-ray range in solar spectrum defining its range from 0.1 to $12.87 \mathrm{~nm}$ with an unprecedented accuracy. Successful identification of X-ray range in solar spectrum prompted to proceed further to accurately identify Bharat Radiation and EUV ranges in solar spectrum.

Hinteregger et al. [8] suggested EUV from 30.0 to $91.0 \mathrm{~nm}$; hence the search for evidence of Bharat Radiation has been confined in between X-ray $(12.87 \mathrm{~nm})$ and EUV $(30.0 \mathrm{~nm})$ ranges. The revised $\mathrm{Rb}$ XRF spectrum in Figure 1(a) shows that the predicted Bharat Radiation begins from $12.87 \mathrm{~nm}$ where $\mathrm{Rb} \mathrm{X}$-ray range 
ends. Excellently agreeing with it, the solar spectrum in Figure 3 also shows an intense spectral range with a steep rise from $12.87 \mathrm{~nm}$ where X-ray range ends. The next and the most important task has been to determine the other end of Bharat Radiation range. As it was not possible to experimentally measure Bharat Radiation and EUV wavelengths from Rb XRF source in the previous study [6], the minimum EUV wavelength $47.488 \mathrm{~nm}$ ( $\mathrm{Rb}$ II) was quoted from the familiar atomic spectra [17]. Thus, the predicted Bharat Radiation range from $\mathrm{Rb}$ XRF source was expected to be lying somewhere in 12.87 to $47.488 \mathrm{~nm}$ range as shown in Figure 1(a). The intense spectral range rising from $12.87 \mathrm{~nm}$ and steeply falling after a prominent peak at $30.4 \mathrm{~nm}$ in Figure 3 fits into the predicted range providing the first and definite evidence for Bharat Radiation in solar spectrum, while such experimental evidence is yet to come from radioisotopes and XRF sources. Bharat Radiation range has been temporarily set to be 12.87 to $31 \mathrm{~nm}$ on inclusion of the peak at $30.4 \mathrm{~nm}$ in solar spectrum pending accurate estimation in future. The comparison of solar spectrum made here with Rb XRF source should not be misconstrued that solar spectrum is due to Rb XRF source. The XRF source greatly helped to calibrate solar Xray range. Otherwise it is to be understood that Bharat Radiation wavelengths are caused by $\gamma-, X-$, or $\beta$ energies from radioisotopes.

Hinteregger et al. [8] thought that EUV range begins from $30.0 \mathrm{~nm}$. Closely agreeing to it, solar spectrum in Figure 3 displayed intense spectral range rising from around $31 \mathrm{~nm}$ where Bharat Radiation range ended. Since EUV lies immediately next to Bharat Radiation in the revised spectrum of Rb XRF source in Figure 1 (a), the measured wavelengths from 31 to $40 \mathrm{~nm}$ have been identified for the first time as solar EUV range. The end limit $40 \mathrm{~nm}$ for EUV was fixed with reference to Figure 3 as it did not extend beyond $40 \mathrm{~nm}$.

In nutshell, the current study has identified for the first time the three distinct wavelength ranges looking like three mounts in solar spectrum that remained puzzling since the beginning of solar spectroscopy. The successive presence of X-ray (0.092969 to $12.87 \mathrm{~nm}$ ), Bharat Radiation (12.87 to $31 \mathrm{~nm}$ ), and EUV (31 to $40 \mathrm{~nm}$ ) ranges in solar spectrum in Fig.3 holds the key for the previously unknown Sunlight phenomenon. This has led to a clear understanding that solar X-rays and EUV are not independent emissions against what has been believed so far. Since, $\gamma-$, X-, or $\beta$ energy reportedly generates Bharat Radiation from within an excited atom of a radioisotope or XRF source; the presence of Bharat Radiation in solar spectrum holds the key evidence that various solar $\gamma-, X-$, and $\beta$ energies generate Bharat Radiation in 12.87 to $31 \mathrm{~nm}$ range from within the same excited atoms of fission products. Ultimately, Bharat Radiation and EUV wavelengths lying sequentially in solar spectrum in Fig. 3 explain that it is the Bharat Radiation that causes EUV.

\subsection{Solar and Fe lines have no valid links}

A question arises on the need to provide an alternative Sunlight phenomenon when the practice of identifying solar lines with $\mathrm{Fe}, \mathrm{He}$, Ne lines etc is well established since the inception of solar spectroscopy nearly a half a century ago. Since high resolution spectra are currently available from National Institute of Standards and Technology (NIST), USA, a review is made on the validity of traditionally linking solar lines with Fe lines etc. A simple spectral comparison of data available in the literature infers serious discrepancies between solar and Fe lines in terms of wavelengths and intensities.

In order to understand how the concept of linking solar lines with Fe lines etc originated, a review is made on the comparison reported by Fawcett and Gabriel in 1965 between solar spectrum, Zeta Discharge and Iron Metal Spark spectra [19]. Intensity of $17.106 \mathrm{~nm}$ emission has been reported to be nearly the same in both solar (10) and Zeta Discharge (11) spectra, a little more than that of iron Metal Spark $17.107 \mathrm{~nm}$ emission (9). Close agreement of these measured wavelengths and intensities was the reason for believing that solar line emissions represent Fe line emissions. However, validity of these findings is verified from the latest solar spectrum made available by Woods et al. in 2011 reproduced in Fig.3 and the latest Fe spectral data available from NIST [20]. Contrary to the findings of Fawcett and Gabriel, the most intense $17.107 \mathrm{~nm}$ emission in the middle of the solar spectrum in Figure 3 disagrees with $17.1073 \mathrm{~nm}$ (Fe IX) emission mentioned by NIST due to low relative intensity just 120 in $\mathrm{Fe}$ spectrum, yet identified with Fe IX in Figure 3 [20]. Inaccurate measurements made by Fawcett and Gabriel that did not agree with the latest spectral data led to the misinterpretation of their findings.

Furthermore, in terms of wavelength and intensity the second most intense $17.693 \mathrm{~nm}$ emission in the middle of the solar spectrum in Fig.3 disagrees with 17.6904, 17.6928, 17.7172 or 17.7235 (Fe VII) emission due to low intensities of $4,10,9$ and 2 respectively reported by NIST, yet traditionally attributed to Fe VII in Figure 3. Fawcett and Gabriel failed to measure the $17.693 \mathrm{~nm}$ emission, instead measured the nearby solar $17.723 \mathrm{~nm}$ emission, Zeta: $17.722 \mathrm{~nm}$ emission and iron Metal Spark: $17.726 \mathrm{~nm}$ emission with intensities 9, 8 and 8 respectively. Inaccurate measurements showing nearly equal intensities in all the three cases might have led to misinterpretation of their data.

Likewise, intense $18.0401 \mathrm{~nm}$ solar emission in Fig.3 has no corresponding Fe emission, yet traditionally attributed to Fe XI from nearby $18.0407 \mathrm{~nm}$ (Fe XI) emission despite its low intensity 90 (Fig.3). Similarly, in 
terms of wavelength and intensity, the relatively low intense solar $28.415 \mathrm{~nm}$ emission in Fig. 3 has no Fe line at the same wavelength, and also it disagrees with the maximum (1000) intense $28.4164 \mathrm{~nm}$ (Fe XV) emission reported by NIST, yet traditionally attributed to Fe XV. The most intense solar $30.4 \mathrm{~nm}$ emission has no Fe or He line at the same wavelength, yet it has long been thought to be He II line [10,11]. The He II Spectral data shows two nearby lines at 30.378040982 and $30.378581505 \mathrm{~nm}$ but their intensity is not given by NIST, hence it is possible that the solar $30.4 \mathrm{~nm}$ emission may not truly represent He II line. On the basis of thermally excited atomic spectrum only a few solar lines in Figs. 1 to 3 could be identified with Fe lines etc; however majority of solar lines were left out. All these viewpoints thus question the validity of thermal excitation causing the solar optical spectrum as has been believed so far.

On the other hand, likely presence of radioisotopes as fission products on Sun's visible surface that mainly emit UV, over $83 \%$ in the gross light intensity, very well explains abundant solar UV [6]. Although it was not possible for the author to previously measure EUV lines within the limited facilities available then, most likely radioisotopes do emit EUV up to $31 \mathrm{~nm}$. Considering the presence of Bharat Radiation wavelengths in solar spectrum, valence excitation by Bharat Radiation resulting into Sun's emission of a new class of EUV and UV dominant atomic spectra from radioisotopes (fission products) regardless of temperature is imminent. That means Sun's high temperatures are not the cause for solar EUV and UV dominant solar spectrum.

\section{Acknowledgements:}

The author is grateful to Tom Woods, LASP, Univ. of Colorado-Boulder for sending me the full text of his paper and Fig. 3 reported here reproduced form his paper.

\section{References}

[1] M.A.Padmanabha Rao, X-ray source emits not only X-rays but also low energy electromagnetic radiation. Presented in 1998 Symposium on Radiation Measurements and Applications, Ann Arbor, U.S.A., 1998, Abstract 3PW26. http://www.angelfire.com/sc3/1010/michigan1998.html

[2] M.A.Padmanabha Rao, Possible biological effects by UV radiation newly detected from internally administered radioisotopes. in Proceedings of the Symposium on Low Level Electromagnetic Phenomena in Biological Systems (BIOSYS- '99), Eds. Jitendra Behari and Indian Journal of Biochemistry and Biophysics, National Institute of Science Communication, New Delhi-110012, 1999 , p 68. http:/www.angelfire.com/sc3/1010/uvdosimetry.html

[3] M.A.Padmanabha Rao, Discovery of light emission from XRF sources, Presented in 50th Annual Denver Conference, Steamboat Springs, U.S.A. Abstract F-01, p.124. http://www.dxcicdd.com/01/pdf/F-01.pdf

[4] M.A.Padmanabha Rao, Solar X-rays, gamma rays, and electrons cause EUV by a previously unknown atomic phenomenon, in Proceedings of the $7^{\text {th }}$ International Conference on Human Ecology and Nature (HEN2008), Moscow-Ples, Russia, 2008, Moscow Scientific and Industrial Association "Radon", p.45. http://www.angelfire.com/sc3/1010/Solarfission.html

[5] Carlos Austerlitz1 et al., Enhanced Response of the Fricke Solution Doped with Hematoporphyrin Under X-Rays Irradiation, Braz. arch. biol. technol. 51, n.2, Mar./Apr. 2008, 271-279 http://www.scielo.br/pdf/babt/v51n2/a06v51n2.pdf

[6] M.A.Padmanabha Rao, UV dominant optical emission newly detected from radioisotopes and XRF sources, Braz. J. Phy., 40, no 1, 2010, 38-46 http://dx.doi.org/10.1590/S0103-97332010000100007

[7] H. E. Hinteregger, K. R. Damon, L. Heroux, and L. A. Hall, Space Research I, (Ed) H. K. Kallman Bijl, (North Holland Pub. Co., Amsterdam, 1960) 615.

[8] H. E. Hinteregger, Interplanetary Ionization by Solar Extreme Ultraviolet Radiation, Astrophysical Journal, 132, 1960, 801811.http://articles.adsabs.harvard.edu//full/1960ApJ ...132..801H/0000805.000.html

[9] H. Friedman, Ultraviolet and X Rays from the Sun, Annual Review of Astronomy and Astrophysics, 1, 1963, 59-96. http://articles.adsabs.harvard.edu//full/1963ARA\%26A...1...59F/0000068.000.html

[10] H. E. Hinteregger, L. A. Hall, and W. Schweizer, Solar Xuv-Spectrum from $310 \AA$ to $55 \AA$ A Astrophysical Journal, 140, 1964, 319326. http://adsabs.harvard.edu/full/1964ApJ...140..319H

[11] T. N.Woods, et al., New solar extreme-ultraviolet irradiance observations during flares, The Astrophysical Journal, 739, 59, 2011,113. http://iopscience.iop.org/0004-637X/739/2/59/pdf/0004-637X_739_2_59.pdf

[12] M.A.Padmanabha Rao, Room temperature atomic spectra from solid radioisotopes and XRF sources, Presented at the $34^{\text {th }}$ Conference of European Group for Atomic spectroscopy (34thEGAS), Sofia, 2002, F2-4, 103. http://www.angelfire.com/sc3/1010/egas34.html

[13] Margaret West., et al., Atomic spectrometry update-X-ray fluorescence spectrometry, J. Anal. At. Spectrom., 26, 2011, $1919-1963$. DOI: $10.1039 / \mathrm{C} 1 \mathrm{JA} 90038 \mathrm{~B}$

[14] T. N. Woods, et al., XUV Photometer System (XPS): Improved Solar Irradiance Algorithm Using CHIANTI Spectral Models, Solar Phys, 250, 2008, 235-267. DOI 10.1007/s11207-008-9196-6

[15] W. M. Neupert, X Rays from the Sun, Annual Review of Astronomy and Astrophysics, 7, 1969, 121-148. DOI: 10.1146/annurev.aa.07.090169.001005

[16] J. Lilensten, et al., Ann. Geophys., 26, 2008, 269-279. doi:10.5194/angeo-26-269-2008

[17] R.L. David, and H.P.R. Frederikse, C.R.C. Handbook of Chemistry and Physics. $74^{\text {th }}$ Edition (CRC Press Inc, London, 1993-1994)

[18] P. Testa, J.J. Drake, and E. Landi, Testing EUV/X-ray atomic data for the solar dynamics observatory, The Astrophysical Journal, 745:111, 2012 February 1,1-10. doi:10.1088/0004-637X/745/2/111

[19] B. C. Fawcett, and A. H. Gabriel, New Spectra of the Iron Transition Elements of Astrophysical Interest, Astrophysical Journal, vol. 141, 1965ApJ...141..343F, 343-353. http://articles.adsabs.harvard.edu//full/1965ApJ...141..343F/0000344.000.html

[20] Kramida, A., Ralchenko, Yu., Reader, J., and NIST ASD Team (2012). NIST Atomic Spectra Database (ver. 5.0), National Institute of Standards and Technology, Gaithersburg, MD. http://physics.nist.gov/PhysRefData/ASD/lines form.html 